\title{
Discovery of WASP-65b and WASP-75b: Two hot Jupiters without highly inflated radii ${ }^{\star}$
}

Y. Gómez Maqueo Chew ${ }^{1,2,3}$, F. Faedi ${ }^{1}$, D. Pollacco ${ }^{1}$, D. J. A. Brown ${ }^{4}$, A. P. Doyle ${ }^{5}$, A. Collier Cameron ${ }^{4}$, M. Gillon ${ }^{7}$, M. Lendl ${ }^{6}$, B. Smalley ${ }^{5}$, A. H. M. J. Triaud ${ }^{6,8}$, R. G. West ${ }^{1}$, P. J. Wheatley ${ }^{1}$, R. Busuttil ${ }^{13}$, C. Liebig ${ }^{4}$, D. R. Anderson ${ }^{5}$, D. J. Armstrong ${ }^{1}$, S. C. C. Barros ${ }^{9}$, J. Bento ${ }^{1,10}$, J. Bochinski ${ }^{13}$, V. Burwitz ${ }^{14}$, L. Delrez ${ }^{7}$, B. Enoch ${ }^{4}$, A. Fumel ${ }^{7}$, C. A. Haswell ${ }^{13}$, G. Hébrard ${ }^{11,12}$, C. Hellier ${ }^{5}$, S. Holmes ${ }^{13}$, E. Jehin ${ }^{7}$, U. Kolb ${ }^{13}$, P. F. L. Maxted ${ }^{5}$, J. McCormac ${ }^{15,1}$, G. R. M. Miller ${ }^{4}$, A. J. Norton ${ }^{13}$, F. Pepe ${ }^{6}$, D. Queloz ${ }^{6,16}$, J. Rodríguez ${ }^{17}$, D. Ségransan ${ }^{6}$, I. Skillen ${ }^{15}$, K. G. Stassun ${ }^{2,18}$, S. Udry ${ }^{6}$, and C. Watson ${ }^{19}$

1 Department of Physics, University of Warwick, Coventry CV4 7AL, UK e-mail: y.gomez@warwick.ac.uk

2 Physics and Astronomy Department, Vanderbilt University, Nashville, Tennessee 37235, USA

3 Centro de Radioastronomía y Astrofísica, UNAM, Apartado Postal 3-72, 58089 Morelia, Michoacán, México

4 School of Physics and Astronomy, University of St Andrews, St Andrews, Fife KY16 9SS, UK

5 Astrophysics Group, Keele University, Staffordshire, ST5 5BG, UK

6 Observatoire astronomique de l'Université de Genève, 51 ch. des Maillettes, 1290 Sauverny, Switzerland

7 Université de Liège, Allée du 6 août 17, Sart Tilman, Liège 1, 4000 Liège, Belgium

${ }^{8}$ Department of Physics, and Kavli Institute for Astrophysics and Space Research, Massachusetts Institute of Technology, Cambridge, MA 02139, USA

9 Aix-Marseille Université, CNRS, LAM (Laboratoire d'Astrophysique de Marseille) UMR 7326, 13388 Marseille, France

10 Department of Physics and Astronomy, Macquarie University, NSW 2109 Sydney, Australia

11 Institut d'Astrophysique de Paris, UMR7095 CNRS, Université Pierre \& Marie Curie, 75014 Paris, France

12 Observatoire de Haute-Provence, CNRS/OAMP, 04870 St Michel l'Observatoire, France

13 Department of Physical Sciences, The Open University, Milton Keynes, MK7 6AA, UK

14 Max Planck Institut für Extraterrestrische Physik, Giessenbachstrasse 1, 85748 Garching, Germany

15 Isaac Newton Group of Telescopes, Apartado de Correos 321, 38700 Santa Cruz de Palma, Spain

16 Department of Physics, University of Cambridge, J J Thomson Av, Cambridge, CB3 OHE, UK

17 Observatori Astronòmic de Mallorca, Camí de l’Observatori s/n, 07144 Costitx, Mallorca, Spain

18 Department of Physics, Fisk University, Nashville, Tennessee 37208, USA

19 Astrophysics Research Centre, Queen's University Belfast, University Road, Belfast BT7 1NN, UK

Received 18 July 2013 / Accepted 27 September 2013

\section{ABSTRACT}

\begin{abstract}
We report the discovery of two transiting hot Jupiters, WASP-65b $\left(M_{\mathrm{pl}}=1.55 \pm 0.16 M_{\mathrm{J}} ; R_{\mathrm{pl}}=1.11 \pm 0.06 R_{\mathrm{J}}\right)$, and WASP-75b $\left(M_{\mathrm{pl}}=1.07 \pm 0.05 M_{\mathrm{J}} ; R_{\mathrm{pl}}=1.27 \pm 0.05 R_{\mathrm{J}}\right)$. They orbit their host star every $\sim 2.311$, and $\sim 2.484$ days, respectively. The planet host WASP-65 is a G6 star $\left(T_{\text {eff }}=5600 \mathrm{~K},[\mathrm{Fe} / \mathrm{H}]=-0.07 \pm 0.07\right.$, age $\left.\gtrsim 8 \mathrm{Gyr}\right)$; WASP-75 is an F9 star $\left(T_{\text {eff }}=6100 \mathrm{~K},[\mathrm{Fe} / \mathrm{H}]=0.07 \pm\right.$ 0.09 , age $\sim 3 \mathrm{Gyr})$. WASP-65b is one of the densest known exoplanets in the mass range 0.1 and $2.0 M_{\mathrm{J}}\left(\rho_{\mathrm{pl}}=1.13 \pm 0.08 \rho_{\mathrm{J}}\right)$, a mass range where a large fraction of planets are found to be inflated with respect to theoretical planet models. WASP-65b is one of only a handful of planets with masses of $\sim 1.5 \mathrm{M}_{\mathrm{J}}$, a mass regime surprisingly underrepresented among the currently known hot Jupiters. The radius of WASP-75b is slightly inflated $(\$ 10 \%)$ as compared to theoretical planet models with no core, and has a density similar to that of Saturn $\left(\rho_{\mathrm{pl}}=0.52 \pm 0.06 \rho_{\mathrm{J}}\right)$.
\end{abstract}

Key words. planetary systems - stars: individual: WASP-65 - stars: individual: WASP-75

\section{Introduction}

Since the discovery of the first extrasolar planet around a mainsequence star, 51 Peg (Mayor \& Queloz 1995), our understanding of planetary systems has dramatically evolved. Planetary science, which was previously based solely on our own Solar

* Light curves are only available at the CDS via anonymous ftp to cdsarc.u-strasbg.fr (130.79.128.5) or via http://cdsarc.u-strasbg.fr/viz-bin/qcat?]/A+A/559/A36
System, must be able to explain the observed diversity in physical properties and trends in the known exoplanet population (e.g., Baraffe et al. 2010; Cameron 2011). An exceptionally valuable subset of the known extrasolar planets are those that transit the disc of their host star. To date, there are over 300 confirmed transiting exoplanets in the literature ${ }^{1}$. Most of these discoveries have been the product of ground-based surveys, of which the Wide Angle Search for Planets (WASP; Pollacco et al. 2006) has

\footnotetext{
See http://exoplanet.eu/
} 
been the most successful, along with the HATNet Project (Bakos et al. 2004), OGLE-III (Udalski et al. 2002), TrES (Alonso et al. 2004), XO Project (McCullough et al. 2005), and KELT (Pepper et al. 2007). The space missions CoRoT (Baglin et al. 2006), and Kepler (Borucki et al. 2010) have also significantly increased the number of discovered transiting planetary systems, and have been able to find much smaller planets than those that have been discovered from the ground, as well as multi-planetary and circumbinary transiting systems.

With knowledge of the physical properties of the stellar host, the transisting system's particular orbital geometry allows us to measure both the actual mass (i.e., $M_{\mathrm{pl}}$ instead of $M_{\mathrm{pl}} \sin i$ ) and the radius of the transiting planet (e.g., Charbonneau et al. 2000). The wide range of observed planetary radii and, in particular, the large fraction of close-in Jupiter-mass planets with anomalously bloated radii (e.g., Fortney \& Nettelmann 2010; Leconte et al. 2010; Laughlin et al. 2011) challenge planetary structure models. Transiting planets allow us to probe the planetary structure by inferring the bulk composition of the planet from its mean density. For example, among the most bloated planets, WASP-17b (Anderson et al. 2010), and HATP-32b (Hartman et al. 2011) have mean planet densities $\rho_{\mathrm{pl}}$ of 0.06 , and $0.11 \rho_{\mathrm{J}}$, respectively, that are not able to be reproduced with standard core-less planet models which predict the largest planets for a given mass (e.g., Baraffe et al. 2008; Fortney et al. 2007). Thus, planetary inflation mechanisms, such as stellar irradiation (Guillot et al. 1996), atmospheric circulation (e.g., Showman \& Guillot 2002; Guillot et al. 2006), tidal effects (e.g., Bodenheimer et al. 2000; Jackson et al. 2008), enhanced atmospheric opacities (Burrows et al. 2007), and ohmic heating (Batygin \& Stevenson 2010; Wu \& Lithwick 2013) have been proposed to explain these anomalously large radii (see also Baraffe et al. 2010). However, a single mechanism has not been able to explain the entire range of observed radii, and it is possible that a combination of them come into play, with some being more effective than others in differing environments/conditions. Thus, it is paramount to expand the sample of well-characterized transiting planets in order to understand the physical and environmental factors that determine the surprising diversity in planetary radii and orbits that have been thus far discovered.

In this paper, we present two newly identified transiting planets in the WASP Survey: 1SWASP J085317.82+083122.8, hereafter WASP-65; and 1SWASP J224932.56-104031.8, hereafter WASP-75. The WASP discovery photometry is described in Sect. 2.1. Section 2.2 describes the spectroscopic follow-up observations that are used to determine the radial velocities of the planet hosts and the spectroscopically determined stellar parameters. The high-cadence, follow-up photometry, detailed in Sect. 2.3, includes data from four different facilities. We derive the stellar physical properties in Sects. 3.1 and 3.2, and the planetary properties via the simultaneous modelling of the radial velocities and the light curves, and the use of theoretical isochrones in Sect. 3.3. Finally, in Sect. 4, we discuss the implications of these new discoveries in the context of the known planetary population.

\section{Observations}

WASP-65 and WASP-75 have been identified in several allsky catalogues which provide broad-band optical and infrared photometry, as well as proper motion information. Coordinates, broad-band magnitudes and proper motion of the stars are taken from the Fourth US Naval Observatory CCD Astrograph Catalog (UCAC4; Zacharias et al. 2012), and are given in Table 1.
Table 1. Photometric and astrometric properties of the two stars WASP-65 and WASP-75.

\begin{tabular}{lcc}
\hline \hline Parameter & WASP-65 & WASP-75 \\
\hline RA(J2000) & $08: 53: 17.83$ & $22: 49: 32.57$ \\
Dec(J2000) & $+08: 31: 22.8$ & $-10: 40: 32.0$ \\
$B$ & $12.57 \pm 0.01$ & $12.05 \pm 0.03$ \\
$V$ & $11.90 \pm 0.04$ & $11.45 \pm 0.01$ \\
$r$ & $11.72 \pm 0.04$ & $11.33 \pm 0.02$ \\
$i$ & $11.57 \pm 0.01$ & $11.13 \pm 0.07$ \\
$J$ & $10.67 \pm 0.02$ & $10.36 \pm 0.02$ \\
$H$ & $10.44 \pm 0.03$ & $10.10 \pm 0.02$ \\
$K$ & $10.35 \pm 0.02$ & $10.06 \pm 0.03$ \\
$\mu_{\alpha}(\mathrm{mas} / \mathrm{yr})$ & $3.8 \pm 1.3$ & $42.8 \pm 1.9$ \\
$\mu_{\delta}(\mathrm{mas} / \mathrm{yr})$ & $7.1 \pm 1.3$ & $14.7 \pm 1.5$ \\
\hline
\end{tabular}

Notes. The broad-band magnitudes and proper motion are obtained from the UCAC4 catalogue.

\subsection{WASP observations}

The WASP North and South telescopes are located in La Palma (ING - Canary Islands, Spain) and Sutherland (SAAO - South Africa), respectively. Each telescope consists of 8 Canon $200 \mathrm{~mm} f / 1.8$ focal lenses coupled to e2v $2048 \times$ 2048 pixel CCDs, which yield a field of view of $7.8 \times 7.8$ square degrees with a corresponding pixel scale of 13". 7 (Pollacco et al. 2006).

WASP-65 and WASP-75 ( $V=11.90$ and $11.45 \mathrm{mag}$, respectively) are located in an equatorial region of sky that is monitored by both WASP instruments. The WASP observations have an exposure time of $30 \mathrm{~s}$, and a typical cadence of $8 \mathrm{~min}$. All WASP data for the two newly discovered planets were processed with the custom-built reduction pipeline described in Pollacco et al. (2006). The resulting light curves were analysed using our implementation of the Box Least-Squares and SysRem detrending algorithms (see Collier Cameron et al. 2006; Kovács et al. 2002; Tamuz et al. 2005) to search for signatures of planetary transits. Once the targets were identified as planet candidates a series of multi-season, multi-camera analyses were performed to strengthen the candidate detection. In addition, different detrending algorithms (e.g., Kovács et al. 2005) were used on the single season and multi-season light curves to confirm the transit signal and the physical parameters of the planet candidate. These additional tests allow a more thorough analysis of the stellar and planetary parameters derived solely from the WASP data and publicly available catalogues (e.g., UCAC4) thus helping in the identification of the best candidates, as well as to reject possible spurious detections.

WASP-65 was first observed on 2009 January 14, and continued to be monitored over the following observing seasons up to 2011 April 24 with the WASP North facility. This resulted in a total of 18922 photometric points. The WASP data shows the periodic dip characteristic of a transiting planetary signal with a period of $P=2.31$ days, a transit duration of $T_{14} \sim 2.6 \mathrm{~h}$, and a transit depth of $\sim 11 \mathrm{mmag}$. The top panel of Fig. 1 shows the discovery WASP photometry phase-folded over the period derived in Sect. 3.3 superimposed on the model light curve.

WASP-75 was observed with both the WASP telescopes from 2008 June 13 to 2010 November 11. The WASP light curve is comprised of 23751 photometric data points. The WASP light curve is shown in Fig. 1 (bottom panel) folded over the identified transit period of $P=2.48$ days, and presents a transit depth of $\sim 10 \mathrm{mmag}$, and a transit duration of $1.91 \mathrm{~h}$. 

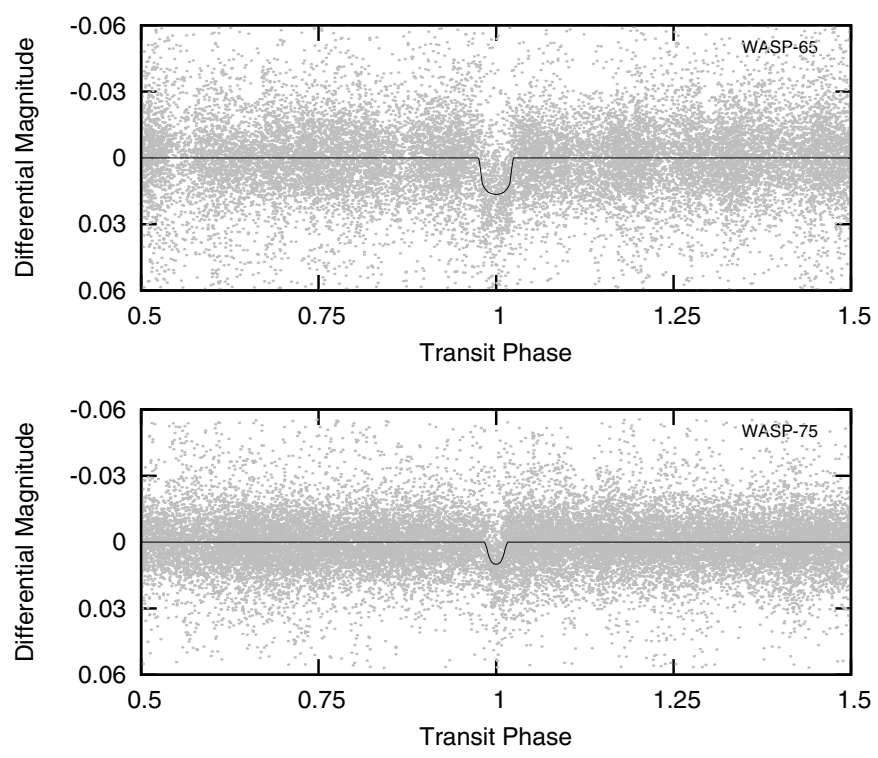

Fig. 1. Discovery WASP light curves. Upper panel: WASP transit light curve of WASP-65b, phase folded on the ephemeris given in Table 7. The black, solid line is the best-fit transit model, as described in Sect. 3.3. Lower panel: same as top panel for WASP-75b light curve.

\subsection{Spectroscopic follow-up observations}

WASP-65 and WASP-75 were observed with the CORALIE spectrograph mounted on the $1.2 \mathrm{~m}$ Euler Swiss telescope at La Silla, Chile (Baranne et al. 1996; Queloz et al. 2000; Pepe et al. 2002). The data were processed with the CORALIE standard data reduction pipeline. The radial velocity uncertainties were derived from the photon noise. All spectra were singlelined. For each planetary system the radial velocities of the host star were computed from a weighted cross-correlation of each spectrum with a numerical mask of spectral type G2, as described in Baranne et al. (1996) and Pepe et al. (2002). To test for possible false-positive scenarios, we performed the crosscorrelation with masks of different stellar spectral types (i.e., G2 and K5) obtaining for each mask similar radial velocity variations.

We present in Tables 2 and 3 the spectroscopic measurements of WASP-65 and WASP-75. Each table contains: the barycentric Julian date $\left(\mathrm{BJD}_{\mathrm{UTC}}-2450000.0\right)$, the stellar radial velocity $(\mathrm{RV})$ measurements $\left(\mathrm{km} \mathrm{s}^{-1}\right)$, the RV uncertainties $\left(\mathrm{km} \mathrm{s}^{-1}\right)$, and the line bisector span measurements $\left(V_{\text {span }} ; \mathrm{km} \mathrm{s}^{-1}\right)$ as defined by Toner \& Gray (1988) and applied to the crosscorrelation function as per Queloz et al. (2001). The RV residuals (in units of $\mathrm{m} \mathrm{s}^{-1}$ ) to the best-fit Keplerian model are found in the last column of Tables 2 and 3; the residuals are calculated to have rms $=12.5 \mathrm{~m} \mathrm{~s}^{-1}$ for WASP-65, and rms $=14.7 \mathrm{~m} \mathrm{~s}^{-1}$ for WASP-75. Figure 2 shows the measured radial velocities and the residuals to the fit folded on the orbital period derived from the simultaneous analysis of the RVs and light curves (see Sect. 3.3) for WASP-65 (left), and WASP-75 (right). Typical errors for the CORALIE RV measurements are $10-15 \mathrm{~m} \mathrm{~s}^{-1}$.

Furthermore, Fig. 3 presents the line bisector variations as a function of time and of measured RV. The line bisector measurements are examined to discard any false-positive scenarios that would reproduce the radial velocity motion of the star mimicking a planet signature that would induce a change in the line profile (e.g., Dall et al. 2006). Any asymmetries in spectral line profiles
Table 2. Radial velocitiy measurements of WASP-65.

\begin{tabular}{lcccc}
\hline \hline $\begin{array}{l}\text { BJD } \\
-2450000\end{array}$ & $\begin{array}{c}R V \\
\left(\mathrm{~km} \mathrm{~s}^{-1}\right)\end{array}$ & $\begin{array}{c}\sigma_{\mathrm{RV}} \\
\left(\mathrm{km} \mathrm{s}^{-1}\right)\end{array}$ & $\begin{array}{c}V_{\text {span }} \\
\left(\mathrm{km} \mathrm{s}^{-1}\right)\end{array}$ & $\begin{array}{c}\mathrm{O}-\mathrm{C} \\
\left(\mathrm{m} \mathrm{s}^{-1}\right)\end{array}$ \\
\hline 5683.53391 & -3.403 & 0.011 & -0.030 & 20 \\
5696.53167 & -2.971 & 0.016 & -0.024 & -9 \\
5706.48433 & -3.361 & 0.014 & -0.033 & 4 \\
5711.48759 & -3.434 & 0.017 & 0.026 & -8 \\
5715.48188 & -3.239 & 0.013 & -0.046 & -21 \\
5721.46162 & -3.028 & 0.018 & 0.024 & 2 \\
5722.46766 & -3.256 & 0.013 & -0.021 & -3 \\
5724.45871 & -3.044 & 0.018 & 0.005 & 1 \\
5725.45819 & -3.404 & 0.013 & -0.045 & -5 \\
5894.84370 & -3.003 & 0.012 & -0.050 & 13 \\
5917.83196 & -3.105 & 0.011 & -0.006 & -17 \\
5926.83061 & -3.234 & 0.011 & -0.028 & 18 \\
5927.86471 & -3.051 & 0.011 & -0.004 & -5 \\
5928.83795 & -3.405 & 0.013 & -0.029 & 2 \\
5958.70536 & -3.443 & 0.012 & 0.007 & -8 \\
6000.56135 & -3.389 & 0.013 & -0.036 & -8 \\
6003.58262 & -2.948 & 0.016 & 0.024 & 24 \\
6004.64137 & -3.350 & 0.017 & -0.035 & 8 \\
\hline
\end{tabular}

Notes. The columns are: the barycentric Julian date, the stellar RV measurements, the RV uncertainties, the line bisector span measurements, and the residuals.

Table 3. Radial velocitiy measurements of WASP-75.

\begin{tabular}{lcccc}
\hline \hline $\begin{array}{l}\text { BJD } \\
-2450000\end{array}$ & $\begin{array}{c}R V \\
\left(\mathrm{~km} \mathrm{~s}^{-1}\right)\end{array}$ & $\begin{array}{c}\sigma_{\mathrm{RV}} \\
\left(\mathrm{km} \mathrm{s}^{-1}\right)\end{array}$ & $\begin{array}{c}V_{\text {span }} \\
\left(\mathrm{km} \mathrm{s}^{-1}\right)\end{array}$ & $\begin{array}{c}\mathrm{O}-\mathrm{C} \\
\left(\mathrm{m} \mathrm{s}^{-1}\right)\end{array}$ \\
\hline 5538.59311 & 2.396 & 0.015 & -0.009 & -10 \\
5795.80716 & 2.107 & 0.012 & 0.039 & -11 \\
5803.56482 & 2.189 & 0.024 & 0.005 & 25 \\
5804.63835 & 2.395 & 0.012 & 0.035 & -5 \\
5809.70786 & 2.378 & 0.010 & -0.043 & -4 \\
5810.76030 & 2.115 & 0.012 & 0.023 & -5 \\
5823.79344 & 2.298 & 0.014 & -0.026 & 14 \\
5830.58703 & 2.110 & 0.011 & 0.006 & -8 \\
5832.60740 & 2.203 & 0.011 & -0.003 & 0 \\
5833.73979 & 2.285 & 0.013 & 0.092 & -2 \\
5855.66998 & 2.173 & 0.012 & 0.029 & 25 \\
5856.67102 & 2.436 & 0.012 & 0.020 & 26 \\
5858.64072 & 2.308 & 0.018 & 0.044 & 0 \\
5883.62851 & 2.354 & 0.019 & -0.036 & -1 \\
5888.58231 & 2.328 & 0.012 & 0.018 & -23 \\
\hline
\end{tabular}

Notes. The columns are: the barycentric Julian date, the stellar RV measurements, the RV uncertainties, the line bisector span measurements, and the residuals.

would be identified by the variation of the line bisector span, and could result from unresolved binarity or from stellar activity. Such effects would cause the bisector spans to vary in phase with radial velocity. No significant correlation is observed between either radial velocity and the line bisector (with a correlation coefficient of 0.04 for WASP-65, and -0.07 for WASP-75), or the bisector and the time at which observations were taken (with a correlation coefficient of $4 \mathrm{e}-06$ for WASP-65, and 6e05 for WASP-75). This supports our conclusion that each signal originates from a planetary companion as opposed to a blended eclipsing binary system, or to stellar activity (e.g., Queloz et al. 2001). 

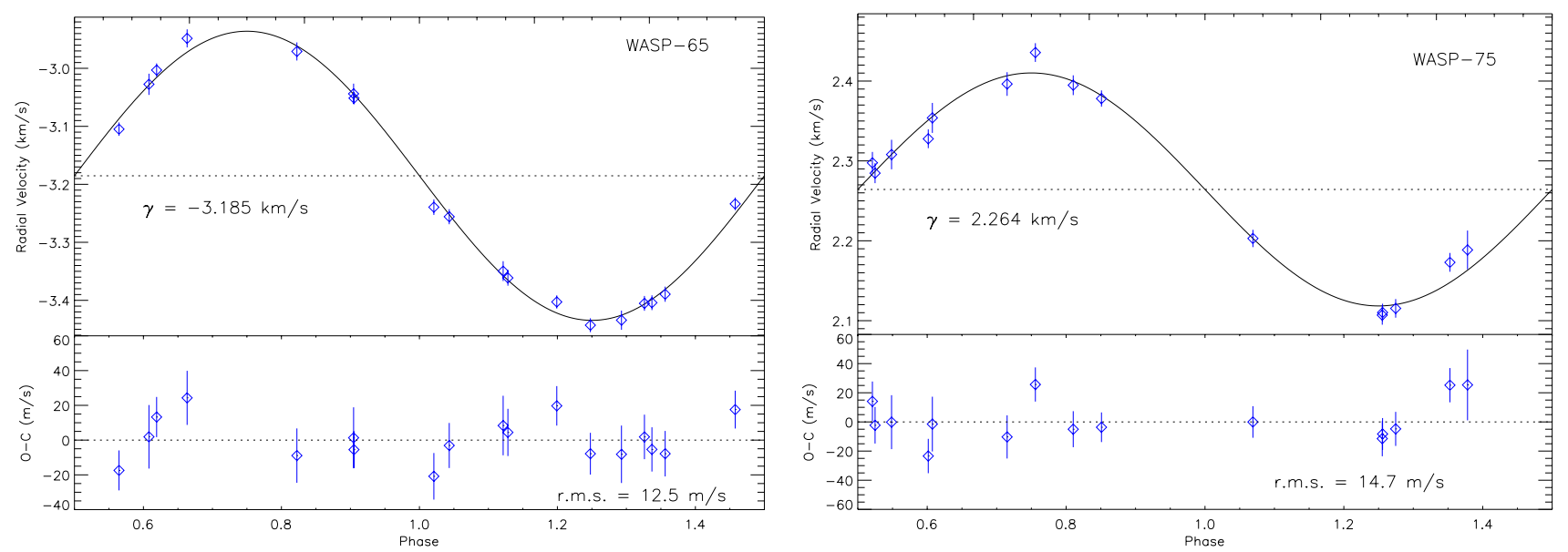

Fig. 2. Upper panels: phase folded radial velocity measurements of WASP-65 (left) and WASP-75 (right) obtained with the CORALIE spectrograph. Superimposed is the best-fit model RV curve with parameters from Table 7. The centre-of-mass velocity is marked by the horizontal dotted line. Lower panels: residuals from the radial velocity fit plotted against orbital phase; the dotted line in the lower panels marks zero. The residuals are in units of $\mathrm{m} \mathrm{s}^{-1}$.
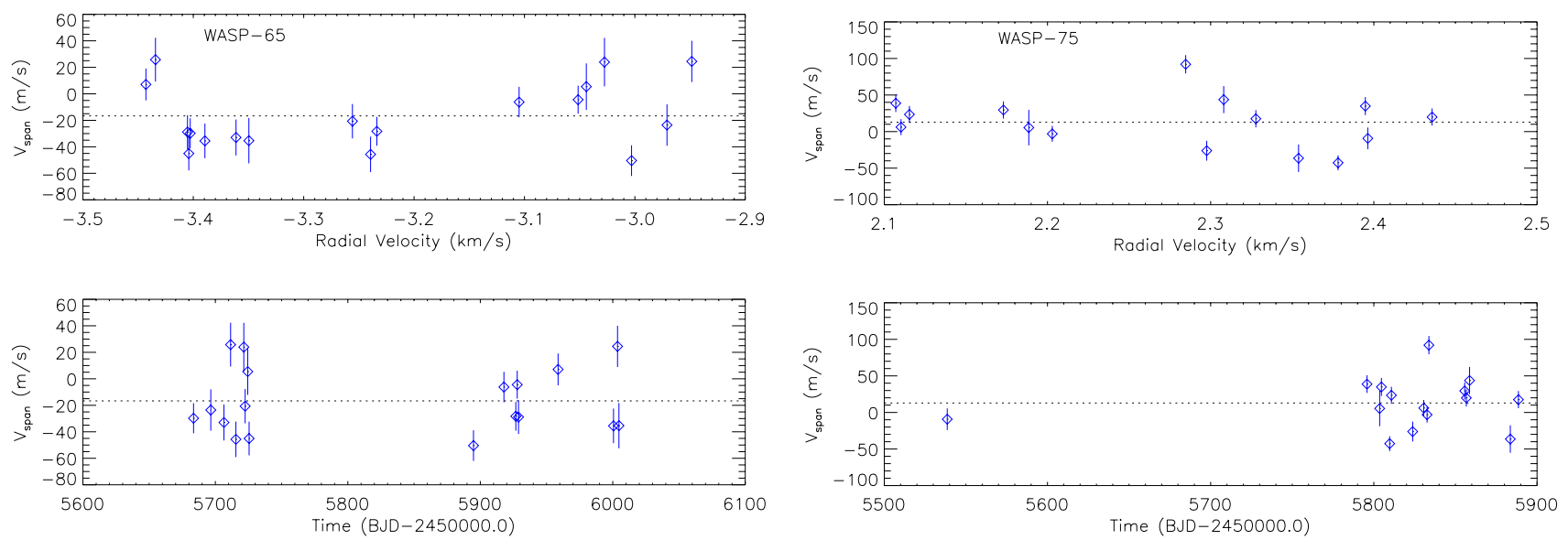

Fig. 3. Upper panels: bisector span measurements of WASP-65 (left) and WASP-75 (right) as a function of radial velocity. The horizontal dotted line marks the mean line bisector span $\left\langle V_{\text {span }}\right\rangle=-0.017 \mathrm{~km} \mathrm{~s}^{-1}$, and $\left\langle V_{\text {span }}\right\rangle=0.013 \mathrm{~km} \mathrm{~s}^{-1}$, respectively. Lower panels: bisector span measurements as a function of time (BJD $\left.\mathrm{BTC}_{\mathrm{UT}}-2450000.0\right)$ for WASP-65 (left) and WASP-75 (right). The bisector spans of both planet hosts are of the same order of magnitude as the errors in the radial velocity measurements, and show no significant variation nor correlation with radial velocity or time. This suggests that the radial velocity variations (with semi-amplitudes of $K_{1}=0.249 \pm 0.005 \mathrm{~km} \mathrm{~s}^{-1}$ for WASP-65b, and $K_{1}=0.146 \pm 0.004 \mathrm{~km} \mathrm{~s}^{-1}$ for WASP-75b) are due to Doppler shifts of the stellar lines induced by a planetary companion rather than stellar profile variations due to stellar activity or a blended eclipsing binary.

\subsection{Follow-up multi-band photometry}

In order to better constrain the systems' parameters, highcadence, high-precision time series photometry during the transits of WASP-65b and WASP-75b were obtained. These follow-up light curves include data from four different telescopes (see below for details), and are summarized in Table 4. All photometric data presented here are available electronically from CDS. We show in Figs. 4 and 5 the follow-up photometry for the transits of WASP-65b, and WASP-75b, respectively. In each plot we show the differential magnitude versus orbital phase, along with the residual to the best-fit model (see Sect. 3.3). The data are phase folded on the ephemeris derived by our analysis of each individual object as given in Table 7.

\subsubsection{TRAPPIST observations}

Both WASP systems were observed with TRAPPIST (TRAnsiting Planets and PlanetesImals Small Telescope; Gillon et al. 2011) located at ESO La Silla, Chile. Its
Table 4. Follow-up time-series transit photometry.

\begin{tabular}{lcll}
\hline \hline Planet & Date & Instrument & Filter \\
\hline \multirow{4}{*}{ WASP-65b } & 20111222 & TRAPPIST & $I+z$ \\
& 20120105 & EulerCam & Gunn $r$ \\
& 20120119 & JGT & $R_{\mathrm{C}}$ \\
& 20120218 & TRAPPIST & blue blocking \\
& 20130310 & PIRATE & $R$ \\
\hline \multirow{4}{*}{ WASP-75b } & 20111123 & TRAPPIST & $I+z$ \\
& 20111128 & TRAPPIST & $I+z$ \\
& 20120828 & EulerCam & Gunn $r$ \\
\hline
\end{tabular}

thermoelectrically-cooled camera is equipped with a $2 \mathrm{~K} \times 2 \mathrm{~K}$ Fairchild $3041 \mathrm{CCD}$ with a $22^{\prime} \times 22^{\prime}$ field of view (i.e., $0.65^{\prime \prime} /$ pixel). The observations were done using a readout mode of $2 \times 2 \mathrm{MHz}$ with $1 \times 1$ binning, resulting in a 


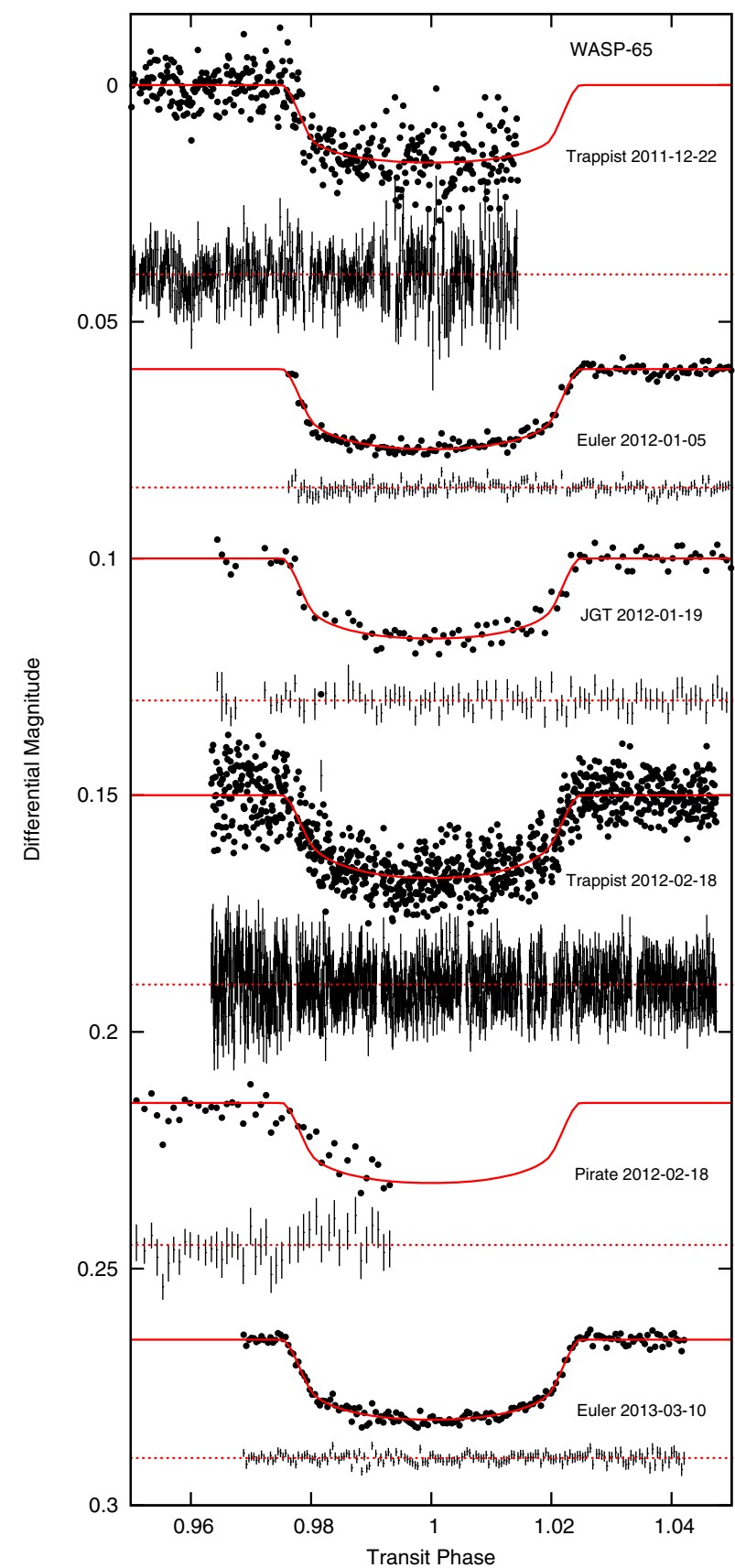

Fig. 4. Follow-up, high-precision, time-series photometry of the WASP-65b during the transit (see Table 4). The observations are shown as black points and are phase folded on the ephemeris shown in Table 7. The superimposed, solid, red line is our best-fit transit model (Sect. 3.3) using the formalism of Mandel \& Agol (2002). The residuals from the fit and the individual data points photometric uncertainties are displayed directly under each light curve. The light curves and residuals are displaced from zero for clarity.

readout + overhead time of $6.1 \mathrm{~s}$ and a readout noise of $13.5 \mathrm{e}^{-}$. A slight defocus was applied to the telescope to optimize the observation efficiency and to minimize pixel-to-pixel effects. The TRAPPIST facility is described in detail by Jehin et al. (2011) and Gillon et al. (2011). A standard pre-reduction (bias, dark, flatfield correction), was carried out and the stellar fluxes were extracted from the images using the IRAF/DAOPHOT aperture photometry software (Stetson 1987). After a careful

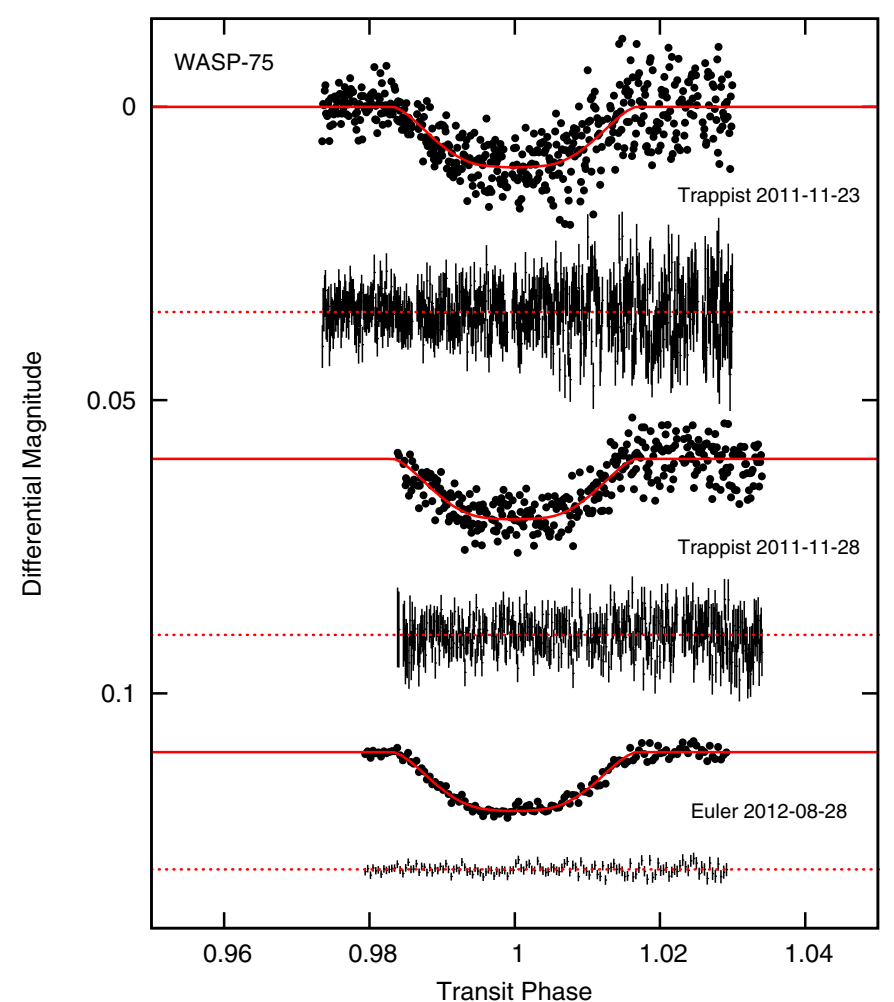

Fig. 5. Follow-up, high signal-to-noise light curves of WASP-75b during transit. Same as Fig. 4.

selection of reference stars, differential photometry was then obtained.

The transit of WASP-65b was observed twice with TRAPPIST. One partial transit was observed on 2011 December 22 in the " $I+z$ " filter (that has a transmittance $>90 \%$ from $750 \mathrm{~nm}$ to beyond $1100 \mathrm{~nm}$ ) with the observations starting about two hours before the transit's ingress, and ending at sunrise after mid-transit but before the start of the egress. As WASP-65 changed position in the night sky, the telescope had to undergo a "meridian flip" which caused the field of view to rotate and the stars to change pixel position. We have accounted for this in our light curve analysis by treating the data before the "meridian flip" as independent from the data after the "meridian flip" to allow for an offset. The exposure time of these data was $20 \mathrm{~s}$ per frame with an average FWHM of 4 pixels for the stellar sources. A full transit of WASP-65b was observed on 2012 February 18 with the blue-blocking filter (with a transmittance $>90 \%$ above $500 \mathrm{~nm}$ ). The observations began more than $0.5 \mathrm{~h}$ before the ingress of the transit and ended over $1 \mathrm{~h}$ after egress. The exposure time per frame was $8.0 \mathrm{~s}$ with an average FWHM of 4.4 pixels. There was also a "meridian flip" during the observations of this transit.

The WASP-75 system was also observed with TRAPPIST during the transit of the planet using the $I+z$ filter. The first TRAPPIST light curve was acquired on 2011 November 23 spanning the full transit, including $\sim 0.5 \mathrm{~h}$ before the transit ingress and $\sim 1 \mathrm{~h}$ after egress. However, the last half hour of data was obtained with an airmass of more than 1.8. The exposure time per frame was $15 \mathrm{~s}$, with an average FWHM of 4.5 pixels. WASP-75 was observed again with TRAPPIST on 2011 November 28, obtaining photometry of an almost full transit. The observations began shortly after ingress started and ended about $1 \mathrm{~h}$ after egress. The exposure time per frame was $20 \mathrm{~s}$, with an average FWHM of 4.4 pixels. 


\subsubsection{EulerCam observations}

We observed two transits of WASP-65b and one transit of WASP-75b using an r'-Gunn filter with EulerCam which is mounted on the $1.2 \mathrm{~m}$ Euler Swiss telescope at the La Silla Observatory in Chile. All images were corrected for bias and flat-field variations, and the light curves were obtained using differential aperture photometry. Lendl et al. (2012) describe in detail the EulerCam instrument, and the reduction procedures used.

The first observations of WASP-65b captured an almost complete transit (starting shortly after the beginning of the ingress) on 2012 January 06 (UT), using fixed exposure times of $60 \mathrm{~s}$. The second set of observations took place on $2013 \mathrm{March}$ 11 (UT) covering a complete transit, and fixed exposure times of $70 \mathrm{~s}$ were used. In both instances, the detector was read out through four ports in order to improve our efficiency, and the telescope was slightly defocussed to improve the PSF sampling.

A full transit of WASP-75b was observed with EulerCam with the r'-Gunn filter on 2012 August 28 (UT). The observations started about half an hour before the beginning of ingress, and ended about an hour after egress. A similar observing strategy was used as for the WASP-65b transits with the exposure time fixed at $60 \mathrm{~s}$, with defocussing, and the detector readout through four ports.

\subsubsection{JGT observations}

We observed the transit of WASP-65b across its host star with the 1-m James Gregory telescope (JGT) at the University of St Andrews Observatory in Scotland, UK. More details of the telescope and instrument can be found in, e.g., Collier Cameron et al. (2010) and Hebrard et al. (2013). A full transit was observed on 2012 January 19. The JGT light curve is comprised of 99 photometric measurements made using an $R_{\mathrm{C}}$ (Cousins) filter with an exposure time of $150 \mathrm{~s}$ each. The pre-eclipse out-oftransit measurements and part of ingress were affected by clouds, but the full width of transit, including times of first and last contact, were detected. The stellar fluxes were extracted from the flat-fielded CCD frames using the photometry routines in the Starlink PHOTOM package. The differential photometry was calculated using a single nearby comparison star, which was the only object brighter than the target in the $15^{\prime}$ field of view.

\subsubsection{PIRATE observations}

The transit of WASP-65b was also observed on 2012 February 18 by the PIRATE Facility ${ }^{2}$ (PIRATE Mk II configuration) located at the Observatori Astronòmic de Mallorca (for details see Holmes et al. 2011). The time series is composed of 120-s exposures that captured nearly $6 \mathrm{~h}$ of out-of-transit light curve (with the pier flip after about $2 \mathrm{~h}$ ), as well as the ingress. The conditions were good, giving a typical FWHM of 3.1". Additional out-of-transit and in-transit data were obtained with non-optimal weather conditions on 2012 January 19, 2012 March 02, and 2012 March 03 and were only used to constrain the ephemeris of the transit. All frames were taken with the Baader $R$ filter; this has a performance similar to the Astrodon Sloan $r^{\prime}$ filter used by the APASS survey (Smith et al. 2010). The data sets were calibrated in the standard way using flat field, dark and bias frames. We constructed the light curves using the ensemble photometry pipeline described in Holmes et al. (2011). Pre- and post-pier flip branches were analysed separately.

\footnotetext{
2 http://pirate.open.ac.uk/index.html
}

Table 5. Stellar properties of WASP-65, and WASP-75 from spectroscopic analysis.

\begin{tabular}{lcc}
\hline \hline Parameter & WASP-65 & WASP-75 \\
\hline$T_{\text {eff }}(\mathrm{K})$ & $5600 \pm 100 \mathrm{~K}$ & $6100 \pm 100 \mathrm{~K}$ \\
$\log g$ & $4.25 \pm 0.1$ & $4.5 \pm 0.1$ \\
$\xi_{\mathrm{t}}\left(\mathrm{km} \mathrm{s}^{-1}\right)$ & $0.9 \pm 0.1$ & $1.3 \pm 0.1$ \\
$v \sin i_{\star}\left(\mathrm{km} \mathrm{s}^{-1}\right)$ & $3.6 \pm 0.5$ & $4.3 \pm 0.8$ \\
{$[\mathrm{Fe} / \mathrm{H}]$} & $-0.07 \pm 0.07$ & $0.07 \pm 0.09$ \\
{$[\mathrm{Na} / \mathrm{H}]$} & $0.08 \pm 0.13$ & $0.14 \pm 0.05$ \\
{$[\mathrm{Mg} / \mathrm{H}]$} & $0.07 \pm 0.08$ & $0.17 \pm 0.15$ \\
{$[\mathrm{Si} / \mathrm{H}]$} & $0.23 \pm 0.06$ & $0.10 \pm 0.09$ \\
{$[\mathrm{Ca} / \mathrm{H}]$} & $0.10 \pm 0.15$ & $0.11 \pm 0.09$ \\
{$[\mathrm{Sc} / \mathrm{H}]$} & $0.07 \pm 0.10$ & $0.19 \pm 0.08$ \\
{$[\mathrm{Ti} / \mathrm{H}]$} & $0.04 \pm 0.07$ & $0.10 \pm 0.13$ \\
{$[\mathrm{~V} / \mathrm{H}]$} & $0.05 \pm 0.11$ & $0.13 \pm 0.09$ \\
{$[\mathrm{Cr} / \mathrm{H}]$} & $0.04 \pm 0.13$ & $0.10 \pm 0.10$ \\
{$[\mathrm{Co} / \mathrm{H}]$} & $0.14 \pm 0.10$ & $0.15 \pm 0.10$ \\
{$[\mathrm{Ni} / \mathrm{H}]$} & $0.05 \pm 0.06$ & $0.08 \pm 0.10$ \\
$\log A(\mathrm{Li})$ & $<1.14 \pm 0.10$ & $2.52 \pm 0.09$ \\
$\mathrm{Sp} . \mathrm{type}$ & $\mathrm{G} 6$ & $\mathrm{~F} 9$ \\
$\mathrm{Distance}(\mathrm{pc})$ & $310 \pm 50$ & $260 \pm 70$ \\
\hline
\end{tabular}

Notes. Spectral type estimated from $T_{\text {eff }}$ using the table in Gray (2008).

\section{Results}

\subsection{Spectroscopically-determined stellar properties}

For both planets the same stellar spectral analysis has been performed by co-adding individual CORALIE spectra. The standard pipeline reduction products were used in the analysis. The analysis was performed using the methods given in Gillon et al. (2009). The $\mathrm{H}_{\alpha}$ line was used to determine the effective temperature $\left(T_{\text {eff }}\right)$. The surface gravity ( $\left.\log g\right)$ was determined from the Ca I lines at $6122 \AA, 6162 \AA$ and $6439 \AA$ (Bruntt et al. 2010b), along with the $\mathrm{Na} I \mathrm{D}$ and $\mathrm{Mg}$ I $b$ lines. The parameters for WASP-65 and WASP-75 obtained from the spectral analysis are listed in Table 5.

The elemental abundances were determined from equivalent width measurements of several clean and unblended lines. A value for microturbulence $\left(\xi_{\mathrm{t}}\right)$ was determined from Fe I using the method of Magain (1984). The quoted errors are estimated to include the uncertainties in $T_{\text {eff }}, \log g$ and $\xi_{\mathrm{t}}$, as well as the scatter due to measurement (dependent on data quality), and atomic data uncertainties. The projected stellar rotation velocity $\left(v \sin i_{\star}\right)$ was determined by fitting the profiles of several unblended Fe I lines. For each system, the macroturbulence $\left(v_{\text {mac }}\right)$ was assumed based on the calibration by Bruntt et al. (2010a). The telluric lines around $6300 \AA$ were used to determine the instrumental FWHM. There are no emission peaks evident in the $\mathrm{Ca}$ II $\mathrm{H}+\mathrm{K}$ lines in the spectra of the two planet hosts. The parameters obtained for each planet host from the spectroscopic analysis are discussed below:

WASP-65: a total of 10 individual CORALIE spectra of WASP-65 were co-added to produce a single spectrum with a typical $\mathrm{S} / \mathrm{N}$ of around 60:1. Our spectral analysis yields $T_{\text {eff }}=5600 \pm 100 \mathrm{~K}, \log g=4.25 \pm 0.10(\mathrm{cgs})$, and $[\mathrm{Fe} / \mathrm{H}]=-0.07 \pm 0.07 \mathrm{dex}$, and a spectral type of G6V. Taking into account the instrumental line profile $(F W H M=0.11 \pm$ $0.01 \AA)$ and the macroturbulence $\left(v_{\text {mac }}=2.0 \pm 0.3 \mathrm{~km} \mathrm{~s}^{-1}\right)$, 
the projected stellar rotational velocity was determined to be $v \sin i_{\star}=3.6 \pm 0.5 \mathrm{~km} \mathrm{~s}^{-1}$. There is no significant detection of lithium in the spectra, with an equivalent width upper limit of $12 \mathrm{~m} \AA$, corresponding to an abundance upper limit of $\log A(\mathrm{Li})$ $<1.14 \pm 0.10$. This implies an age of at least several Gyr (Sestito $\&$ Randich 2005). The rotation rate $(P=17.9 \pm 3.4 \mathrm{~d})$ implied by the $v \sin i_{\star}$ gives a gyrochronological age of $\sim 1.72_{-0.76}^{+1.26} \mathrm{Gyr}$ using the empirical relationship of Barnes (2007). The latter of these age indicators suggests that WASP-65 is a younger version of our Sun. However, assuming a higher value for $v_{\text {mac }}$, like that from the calibrations of Valenti \& Fischer (2005) or Gray (2008), $v \sin i_{\star}$ would be lower and a longer rotation period would be derived implying an older age for the planet host. A better measure of the rotation period of WASP-65 would be from photometric rotational modulation; none was observable in the WASP light curve, which was searched with a sine-wave fitting algorithm, described in Maxted et al. (2011). Moreover, given the lack of stellar activity (i.e., the absence of $\mathrm{Ca}$ II $\mathrm{H}+\mathrm{K}$ emission) and the comparison of the stellar properties with theoretical evolutionary models (see Table 6), WASP-65 seems to be older than our Sun ( $\gtrsim 8$ Gyr).

WASP-75: a total of 15 individual CORALIE spectra of WASP-75 were co-added to produce a single spectrum with a typical S/N of around 100:1. The derived spectroscopic properties of WASP-75 are $T_{\text {eff }}=6100 \pm 100 \mathrm{~K}, \log g=4.5 \pm 0.1$ $(\mathrm{cgs})$, and $[\mathrm{Fe} / \mathrm{H}]=0.07 \pm 0.09 \mathrm{dex}$, and a spectral type of F9V. Considering a macroturbulence $\left(v_{\mathrm{mac}}=3.5 \pm 0.3 \mathrm{~km} \mathrm{~s}^{-1}\right)$ and an instrumental FWHM of $0.11 \pm 0.01 \AA$, the best fitting value of $v \sin i_{\star}=4.3 \pm 0.8 \mathrm{~km} \mathrm{~s}^{-1}$ was obtained. The lithium line strength $\log A(\mathrm{Li})=2.52 \pm 0.09$ implies an age of approximately 2 5 Gyr according to Sestito \& Randich (2005). The rotation rate $(P=11.7 \pm 2.7 \mathrm{~d})$ implied by the $v \sin i_{\star}$ gives a gyrochronological age of $\sim 1.69_{-0.87}^{+1.58} \mathrm{Gyr}$ using the Barnes (2007) relation. Both the gyrochronological and the age derived from the Li abundance imply that WASP-75 is a relatively young system, which is consistent with the age derived from stellar evolutionary tracks ( 3-4 Gyr; see Table 6).

\subsection{Stellar mass determination}

The absolute properties of the planet are well determined to the extent the stellar physical properties are accurate and precise. Thus, we have determined the stellar mass using four theoretical evolutionary models and a stellar empirical calibration (Enoch et al. 2010; Torres et al. 2010), as described below. The mean of these five independent mass estimates is adopted for the rest of our analysis (see Sect. 3.3), and its uncertainty is given by the possible range of masses including the individual uncertainties. Table 6 summarizes the results from the interpolation of the four theoretical models, from the empirical relationship, and, lastly, the mean stellar mass adopted to derive the final orbital, stellar, and planetary properties.

The stellar mass is derived from the spectroscopicallydetermined stellar effective temperature and metallicity (Sect. 3.1), and the mean stellar density $\rho_{\star}$, directly determined from transit light curves. Transiting planets allow us to measure $\rho_{\star}$ independently from the $T_{\text {eff }}$ determined from the spectrum (assuming $M_{\mathrm{pl}} \ll M_{\star}$; see also Seager \& MallénOrnelas 2003), as well as of theoretical stellar models (e.g., Sozzetti et al. 2007; Hebb et al. 2009). We measured the mean stellar density of both planet hosts via our Markov-Chain Monte Carlo (MCMC) analysis (see Sect. 3.3). In the case of the theoretical evolutionary models, the four sets of tracks
Table 6. Stellar masses and ages for WASP-65 and WASP-75

\begin{tabular}{|c|c|c|c|c|}
\hline & \multicolumn{2}{|c|}{ WASP-65 } & \multicolumn{2}{|c|}{ WASP-75 } \\
\hline & \multicolumn{4}{|c|}{ Theoretical evolutionary models } \\
\hline & $M_{\star}\left(M_{\odot}\right)$ & Age (Gyr) & $M_{\star}\left(M_{\odot}\right)$ & Age (Gyr) \\
\hline Padova $^{1}$ & $0.89_{-0.02}^{+0.16}$ & $8.9_{-2.3}^{+3.7}$ & $1.14_{-0.04}^{+0.04}$ & $3.01_{-1.08}^{+1.33}$ \\
\hline $\mathrm{YY}^{2}$ & $0.93_{-0.06}^{+0.06}$ & $8.8_{-2.9}^{+3.2}$ & $1.17_{-0.03}^{+0.04}$ & $3.12_{-0.95}^{+0.78}$ \\
\hline Teramo $^{3}$ & $0.95_{-0.18}^{+0.05}$ & $12.2_{-3.2}^{+3.5}$ & $1.12_{-0.04}^{+0.07}$ & $4.32_{-1.49}^{+1.63}$ \\
\hline \multirow[t]{3}{*}{ VRSS $^{4}$} & $0.90_{-0.04}^{+0.07}$ & $11.2_{-3.8}^{+4.5}$ & $1.13_{-0.05}^{+0.07}$ & $3.45_{-0.84}^{+1.66}$ \\
\hline & \multicolumn{4}{|c|}{ Empirical relationship } \\
\hline & \multicolumn{2}{|c|}{$M_{\star}\left(M_{\odot}\right)$} & \multicolumn{2}{|c|}{$M_{\star}\left(M_{\odot}\right)$} \\
\hline \multirow[t]{3}{*}{ Enoch $^{5}$} & \multicolumn{2}{|c|}{$0.99 \pm 0.02$} & \multicolumn{2}{|c|}{$1.15 \pm 0.03$} \\
\hline & \multicolumn{4}{|c|}{ Adopted stellar mass } \\
\hline & \multicolumn{2}{|c|}{$M_{\star}\left(M_{\odot}\right)$} & \multicolumn{2}{|c|}{$M_{\star}\left(M_{\odot}\right)$} \\
\hline Mean & \multicolumn{2}{|c|}{$0.93_{-0.16}^{+0.12}$} & \multicolumn{2}{|c|}{$1.14_{-0.06}^{+0.07}$} \\
\hline
\end{tabular}

References. (1) Marigo et al. (2008); Girardi et al. (2010); (2) Demarque et al. (2004); (3) Pietrinferni et al. (2004); (4) VandenBerg et al. (2006); (5) Enoch et al. (2010).

used are: a) the Padova stellar models by Girardi et al. (2000), b) the Yonsei-Yale (YY) models by Demarque et al. (2004), c) the Teramo models of Pietrinferni et al. (2004), and d) the Victoria-Regina stellar models (VRSS) of VandenBerg et al. (2006). The interpolation of the isochrones and mass tracks for the metallicity derived from the spectral analysis is done using a Delaunay triangulation (Delaunay 1934), as implemented by Bernal (1988) and developped by Pál \& Bakos (2006). The errors are derived using the error ellipse from $T_{\text {eff }}$ and $\rho_{\star}{ }^{-1 / 3}$, taking into account the range of values given by the 1- $\sigma$ uncertainties, and the points at 45 degrees between these on the error ellipse. Additionally, we have incorporated the uncertainty in the stellar mass due to the $1-\sigma$ error in $[\mathrm{Fe} / \mathrm{H}]$. The stellar mass is also derived from the empirical calibration of Enoch et al. (2010) adapted for transiting planets with measurable $\rho_{\star}$ from the study by Torres et al. (2010) of eclipsing binary stars with masses and radii known to better than $3 \%$. The uncertainty on the stellar mass derived from the empirical relationship results from our MCMC analysis (Sect. 3.3). The stellar masses derived for WASP-75 from the four sets of stellar evolution models (Table 6) agree very well with each other, and with the stellar mass from the Enoch calibration from our MCMC analysis, within their 1- $\sigma$ uncertainties. In the case of WASP-65, the $M_{\star}$ from the empirical Enoch relationship is consistent within $2-\sigma$ with the masses derived from the theoretical stellar tracks. For a more robust measurement of the stellar mass, we have calculated the mean of the stellar masses derived from the four theoretical models and the empirical Enoch calibration, which is used as a prior in our MCMC analysis below and is used in the determination of the planetary properties. The uncertainty in the adopted stellar masses are given by the $1-\sigma$ range for the individual derivations in order to account for all sources of error discussed above.

\subsection{Planetary physical properties}

The planetary properties were determined via an MCMC analysis which simultaneously models the WASP photometry, the 
Table 7. System parameters of WASP-65 and WASP-75

\begin{tabular}{lccc}
\hline \hline & WASP-65b & WASP-75b & \\
\hline$P$ & $2.3114243 \pm 0.0000015$ & $2.484193 \pm 0.000003$ & $\mathrm{~d}$ \\
$T_{0}{ }^{a}$ & $6110.68772 \pm 0.00015$ & $6016.2669 \pm 0.0003$ & $\mathrm{~d}$ \\
$T_{14}{ }^{b}$ & $0.11396 \pm 0.00045$ & $0.0822 \pm 0.0011$ & $\mathrm{~d}$ \\
$T_{12}=T_{34}$ & $0.0118_{-0.0003}^{+0.000}$ & $0.030_{-0.002}^{+0.003}$ & $\mathrm{~d}$ \\
$\Delta F=R_{\mathrm{pl}}{ }^{2} R_{\star}$ & $0.01280 \pm 0.00015$ & $0.0107 \pm 0.0003$ & \\
$b$ & $0.149_{-0.095}^{+0.082}$ & $0.882_{-0.008}^{+0.006}$ & $R_{\star}$ \\
$i$ & $88.8_{-0.7}^{+0.8}$ & $82.0_{-0.2}^{+0.3}$ & $\circ$ \\
$K_{1}$ & $0.249 \pm 0.005$ & $0.146 \pm 0.004$ & $\mathrm{~km} \mathrm{~s}^{-1}$ \\
$\gamma$ & $-3.1853 \pm 0.0009$ & $2.26429 \pm 0.00006$ & $\mathrm{~km} \mathrm{~s}^{-1}$ \\
$e$ & 0. & 0. & $f i x e d$ \\
$M_{\star}$ & $0.93 \pm 0.14$ & $1.14 \pm 0.07$ & $M_{\odot}$ \\
$R_{\star}$ & $1.01 \pm 0.05$ & $1.26 \pm 0.04$ & $R_{\odot}$ \\
$\log g_{\star}$ & $4.40 \pm 0.02$ & $4.29+0.02$ & $\mathrm{cgs}$ \\
$\rho_{\star}$ & $0.91_{-0.04}^{+0.03}$ & $0.56 \pm 0.04$ & $\rho_{\odot}$ \\
$M_{\mathrm{pl}}$ & $1.55 \pm 0.16$ & $1.07 \pm 0.05$ & $M_{\mathrm{J}}$ \\
$R_{\mathrm{pl}}$ & $1.112 \pm 0.059$ & $1.270 \pm 0.048$ & $R_{\mathrm{J}}$ \\
$\log g_{\mathrm{pl}}$ & $3.458_{-0.018}^{+0.014}$ & $3.179_{-0.028}^{+0.033}$ & $\mathrm{cgs}$ \\
$\rho_{\mathrm{pl}}$ & $1.13_{-0.08}^{+0.07}$ & $0.52_{-0.05}^{+0.06}$ & $\rho_{\mathrm{J}}$ \\
$a$ & $0.0334_{-0.0017}^{+0.0016}$ & $0.0375_{-0.0008}^{+0.0007}$ & $\mathrm{AU}$ \\
$T_{\mathrm{eq}}$ & $1480 \pm 10$ & $1710 \pm 20$ & $\mathrm{~K}$ \\
\hline & & &
\end{tabular}

Notes. ${ }^{(a)} \mathrm{BJD}_{\mathrm{TDB}}-2450000.0 .{ }^{(b)} T_{14}$ : time at transit between 1 st and 4 th contact.

follow-up, high-cadence photometry, together with CORALIE radial velocity measurements, as described in detail by Collier Cameron et al. (2007) and Pollacco et al. (2008). The parameters used by the MCMC analysis are: the epoch of mid transit $T_{0}$, the orbital period $P$, the fractional change of flux proportional to the ratio of stellar to planet surface areas $\Delta F=R_{\mathrm{pl}}^{2} / R_{\star}^{2}$, the transit duration $T_{14}$, the impact parameter $b$, the radial velocity semiamplitude $K_{1}$, the stellar host mass $M_{\star}$ calculated in Sect. 3.2, the Lagrangian elements $\sqrt{e} \cos \omega$ and $\sqrt{e} \sin \omega$ (where $e$ is the eccentricity and $\omega$ the longitude of periastron), and the systemic or centre-of-mass velocity $\gamma$. For the treatment of the stellar limb-darkening, the 4-coefficient model of Claret (2000, 2004) was adopted, using the corresponding tabulated coefficients for each passband, and the stellar spectroscopic properties. In the case of the WASP photometry, the $R$-band was used as an approximation. Similarly, the $I$ and $V$-bands were used for the TRAPPIST $I+z$ and blue-blocking filters, respectively. The sum of the $\chi^{2}$ for all input data curves with respect to the models was used as the goodness-of-fit statistic, and each light curve is weighted such that the reduced- $\chi^{2}$ of the best-fit solution is $\sim 1$.

An initial MCMC solution with a linear, long-term trend in the radial velocities was explored for both planetary systems by allowing the systemic velocity to change with time (i.e., $\mathrm{d} \gamma / \mathrm{d} t \neq 0$.). No significant variation of the systemic velocity was found for either planetary system. Thus, the rest of the analyses are done assumming no long-term trend in the radial velocities (i.e., $\mathrm{d} \gamma / \mathrm{d} t=0$.). For each planetary system, four different sets of solutions were considered: (a) a circular solution assuming that the stellar host is on the main sequence, (b) a solution with a free-floating eccentricity and the mass-radius main-sequence constraint, (c) a circular orbit without the mass-radius constraint, and (d) an orbit with eccentricity as a free parameter with no main-sequence constraint.

In the case of both planetary systems, when the eccentricity was left as a free paramenter (with and without the mainsequence constraint), it converged to a small, non-zero value $(e<0.02)$ for all solutions. To assess whether these small eccentricities are real, we performed the F-test proposed by Lucy \& Sweeney (1971, see their Eq. (27)). We find in all instances that the resulting eccentricity is spurious. The orbit could be truly eccentric to the resulting $e$, but the available data are unable to differentiate between that and a circular orbit. Furthermore, the longitude of periastron of these eccentric solutions is close to $90^{\circ}$ or $-90^{\circ}$, which could also indicate a spurious eccentricity detection. Thus, we adopt circular orbits for both WASP-65b and WASP-75b.

Additionally, we assessed whether either planet host required the assumption of the star being on the main sequence. Typically this constraint is needed when the follow-up light curves do not include full transits or do not have the necessary precision to well determine the transit duration, depth, and the system's impact parameter. Both WASP-65 and WASP-75 have high-quality follow-up photometry of full transits. Comparing both sets of solutions with and without the mass-radius main-sequence assumption, the solutions with the main-sequence prior have higher $\chi^{2}$ values than those without. For both planetary systems, both solutions with and without the mass-radius constraint are the same within their 1- $\sigma$ uncertainties; however the solutions 


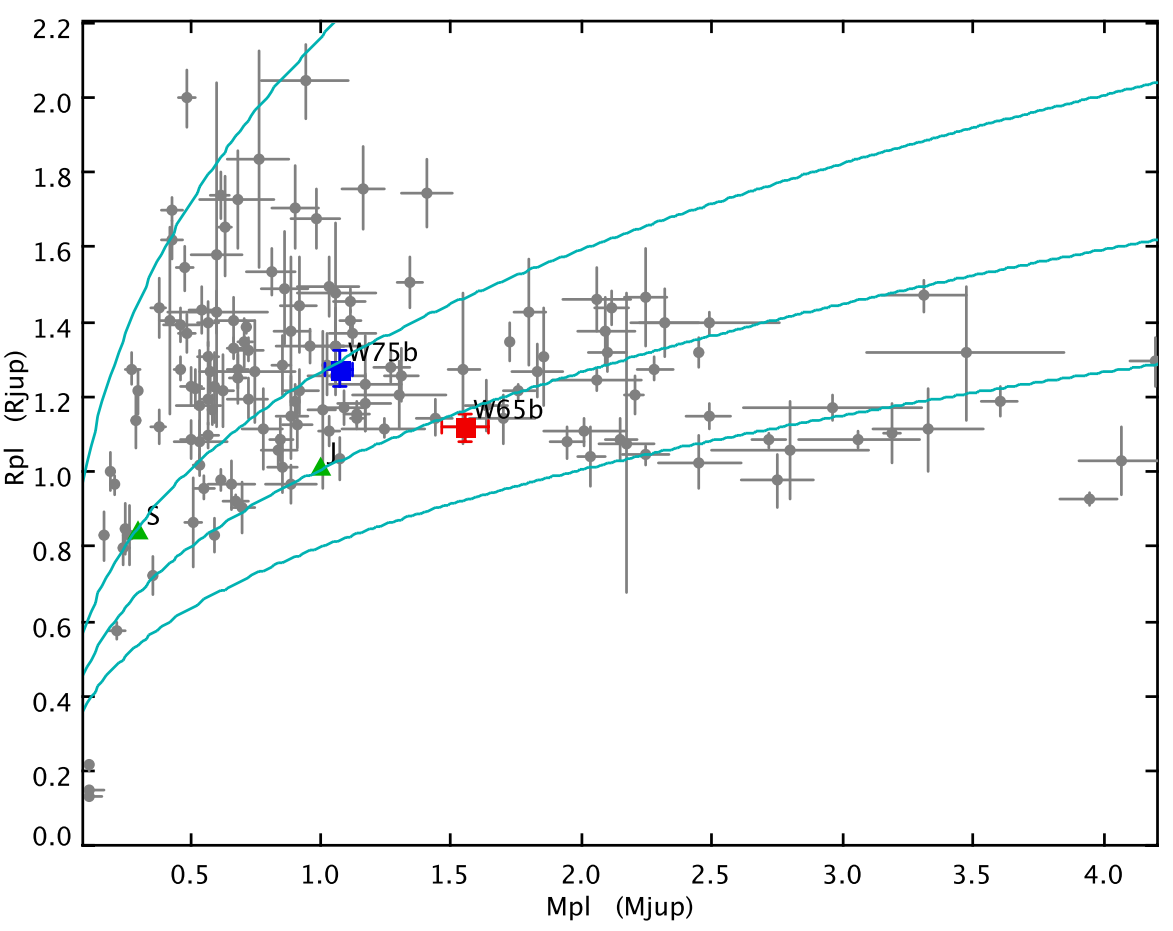

Fig. 6. Planet mass-radius diagram. We present two newly discovered planets from the WASP survey for transiting planets, WASP-65b (redfilled square), and WASP-75b (blue-filled square). The grey points represent the known exoplanets in the Saturn and Jupiter mass regimes taken from exoplanet.org (23 May 2013) and complemented from the literature. Saturn and Jupiter are marked in the greenfilled triangles, and are initialed. The continuous (cyan) lines represent equal density traces of $0.1,0.5,1.0$, and $2.0 \rho_{\mathrm{J}}$, from left to right. WASP-65b lies in between the lower-density giant planets and the higher-density high-mass planets. with the main-sequence constraint have larger uncertainties. Thus, we adopt the solution of each system that does not impose the main-sequence constraint.

Based on the parameters and considerations described above, radius $R$, density $\rho$, and surface gravity $\log g$ of the star (denoted with the subscript $\star$ ) and of the planet (denoted with the subscript ${ }_{\mathrm{pl}}$ ), as well as the mass $M_{\mathrm{pl}}$ and the equilibrium temperature of the planet $T_{\mathrm{eq}}$ are calculated. The planet's equilibrium temperature assumes that it is a black-body $\left(T_{\mathrm{pl}, \mathrm{A}=0}\right)$ and that the energy is efficiently redistributed from the planet's day-side to its night-side. We also calculate the transit ingress (and egress) duration $T_{12}\left(=T_{34}\right)$, and the orbital semi-major axis $a$.

These calculated properties and their $1-\sigma$ uncertainties from our MCMC analysis, adopting circular orbits for both planets and not using the main-sequence constraint, are presented in Table 7. The corresponding best-fit model radial velocity curves are shown in Fig. 2. The transit light curve models are shown in Fig. 1 against the WASP observed photometry of WASP$65 \mathrm{~b}$ (top) and WASP-75b (bottom), in Fig. 4 against the followup transit light curves of WASP-65b, and in Fig. 5 for the WASP-75b follow-up transit light curves. Each figure also contains the individual photometric uncertainties and the residuals to the fit.

\section{Discussion}

We present two newly discovered planets from the WASP survey, WASP-65b and WASP-75b.

In this paper, we have implemented an estimation of the stellar host mass based on both theoretical stellar isochrones (Girardi et al. 2000; Demarque et al. 2004; Pietrinferni et al. 2004; VandenBerg et al. 2006), and the empirical relationship of Torres et al. (2010) as implemented by Enoch et al. (2010) specifically for transiting planets. This allows the inclusion of realistic errors in the host mass determination emcompassing five independent derivations of the stellar mass based on the $\rho_{\star}$ measured from the transit light curve, and the spectroscopicallydetermined $[\mathrm{Fe} / \mathrm{H}]$ and $T_{\text {eff }}$ including their uncertainties, which are not always taken into account. Our analysis includes the propagation of the stellar mass uncertainty in the planet mass and orbital parameters. This is of importance because the planet physical properties are only as accurate as the stellar properties, and any conclusions directly depend on these derived properties.

WASP-65b has a mass of $1.55 \pm 0.16 M_{\mathrm{J}}$, which lies in the mass regime at which Bayliss et al. (2013) identify a surprising lack of known hot Jupiters. Figure 6 shows this scarcity of hot Jupiters at the mass of WASP-65b (red-filled square), and marks the locus in the mass-radius diagram that separates the lowerdensity $\left(\lesssim 1.0 \rho_{\mathrm{J}}\right)$ from the higher-density giant planets $\left(>1.0 \rho_{\mathrm{J}}\right)$. There are four other known planets with masses consistent with that of WASP-65b within their 1- $\sigma$ uncertainties: WASP-5b, WASP-12b, WASP-50b, and WASP-72b. Among these five planets, WASP-65b is the smallest/densest. It is also the one in the orbit with the longest period; though WASP-72b's orbital pe$\operatorname{riod}(\sim 2.22 \mathrm{~d})$ is similar to that of WASP-65b. Their planet hosts range from 5400 (WASP-50) to $6300 \mathrm{~K}$ (WASP-12), and from -0.12 to 0.3 dex in $[\mathrm{Fe} / \mathrm{H}]$. WASP-65 also seems to be the oldest planet host; however, given the uncertainties in the ages, this is not well constrained. It remains unclear whether this paucity of known planets with a mass of $\sim 1.5 M_{\mathrm{J}}$ is the result of a real physical process that might inhibit the formation of giant planets of this mass, a systematic effect in the planetary mass of the known planets because of inaccurate host masses, or due to lownumber statistics. The discovery of WASP-65b suggests that we cannot discard any explanation at this time. More discoveries of hot Jupiters, in tandem with a careful re-analysis of the known transiting planets, such as that proposed by Gómez Maqueo Chew et al. (2013) will enable the confirmation/rejection of these scenarios.

The mean density of WASP-65b $\left(1.13 \pm 0.08 \rho_{\mathrm{J}}\right)$ is slightly higher than that of Jupiter, and in fact, WASP- $65 \mathrm{~b}$ is one of the 
densest planets known in the mass range of $0.1<M_{\mathrm{pl}}<2.0 M_{\mathrm{J}}$. WASP-75b is also shown in Fig. 6, marked by the blue-filled square. With $M_{\mathrm{pl}}=1.07 \pm 0.05 M_{\mathrm{J}}$, WASP-75b has a mean density $\left(0.52 \pm 0.06 \rho_{\mathrm{J}}\right)$ similar to that of Saturn.

Given the measured semi-major axes of their orbits $(0.033 \pm$ $0.002 \mathrm{AU}$ for WASP-65, and $0.0375 \pm 0.0008 \mathrm{AU}$ for WASP-75), we compared the theoretical models of Fortney et al. (2007) for planets orbiting a solar-type star at two different orbital separations ( 0.02 and $0.045 \mathrm{AU})$. We considered the models that predict the largest planet radii: those without a core that are composed of only $\mathrm{H} / \mathrm{He}$, and those with a $10 \mathrm{M}_{\oplus}$ core composed of $50 \%$ rock and $50 \%$ ice and a $\mathrm{H} / \mathrm{He}$ envelope. We find that the radius of WASP-65b $\left(1.11 \pm 0.06 R_{\mathrm{J}}\right)$ is not inflated, and is consistent with all of the predicted radii at $10 \mathrm{Gyr}$ for a $1.5 M_{\mathrm{J}}$ planet in the cases mentioned above. This agreement in the planetary radii could also be considered as evidence of the old age of the system as suggested by the stellar isochrones ( 9-11 Gyr; see Table 6), given that hot Jupiters generally decrease in size as they evolve (see e.g., Fig. 5 in Fortney et al. 2007), independent of heating mechanisms (e.g., Spiegel \& Burrows 2013). In the case of WASP-75b, we find the measured radius of $1.27 \pm 0.05 R_{\mathrm{J}}$ to be inflated by $\$ 10 \%$ as compared to the coreless models for a $1.0 M_{\mathrm{J}}$ planet with an age of $3.16 \mathrm{Gyr}$ orbiting at a distance of 0.02 AU (Fortney et al. 2007).

Perna et al. (2012) identify the equilibrium temperature boundary where the atmospheric heat redistribution starts to be less efficient to be around $\sim 1500-1700 \mathrm{~K}$. WASP-65b $\left(T_{\text {eq }} \sim\right.$ $1500 \mathrm{~K})$ and WASP-75b $\left(T_{\mathrm{eq}} \sim 1700 \mathrm{~K}\right)$ straddle this boundary, and if their atmospheres were observable they could provide insight into the heating/cooling mechanisms of planetary atmospheres. With current capabilities for transmission spectroscopy, it is not possible to study these atmospheres. The upper limit of the atmospheric scale height is given for an atmosphere composed of $100 \%$ molecular hydrogen, and for the case of both WASP-65b and WASP-75b, it is only of a few hundreds of kilometres ( 200 and $\sim 450 \mathrm{~km}$, respectively). In the case of measuring the emission of the planets, the secondary eclipses could be detectable in the $K$-band and in the Spitzer IRAC $1+2$ channels. However, it will make them interesting targets for future planetary atmospheric studies (e.g., JWST and EChO; Gardner et al. 2006; Tinetti et al. 2012).

Acknowledgements. We would like to thank the anonymous referee for significantly improving this paper. The SuperWASP Consortium consists of astronomers primarily from University of Warwick, Queen's University Belfast, St Andrews, Keele, Leicester Universities, The Open University, Isaac Newton Group La Palma and Instituto de Astrofísica de Canarias. The SuperWASP$\mathrm{N}$ camera is hosted by the Issac Newton Group at La Palma and WASPSouth is hosted by SAAO. We are grateful for their support and assistance. Funding for WASP comes from consortium universities and from the UK's Science and Technology Facilities Council. Based on observations made with the CORALIE Echelle spectrograph mounted on the Swiss telescope at ESO La Silla in Chile. TRAPPIST is funded by the Belgian Fund for Scientific Research (Fond National de la Recherche Scientifique, FNRS) under the grant FRFC 2.5.594.09.F, with the participation of the Swiss National Science Fundation (SNF). Y. Gómez Maqueo Chew acknowledges postdoctoral funding support from the Vanderbilt Office of the Provost, through the Vanderbilt Initiative in Data-intensive Astrophysics (VIDA) and through a grant from the Vanderbilt International Office in support of the Vanderbilt-Warwick Exoplanets Collaboration. M. Gillon and E. Jehin are FNRS Research Associates. L. Delrez is a FRIA Ph.D. student of the FNRS. The research leading to these results has received funding from the European Community's Seventh Framework Programme (FP7/20072013) under grant agreement number RG226604 (OPTICON). C. Liebig acknowledges the Qatar Foundation for support from QNRF grant NPRP-09-4761-078. A.H.M.J. Triaud is a Swiss National Science Foundation fellow under grant number PBGEP2-145594.

\section{References}

Alonso, R., Brown, T. M., Torres, G., et al. 2004, ApJ, 613, L153 Anderson, D. R., Hellier, C., Gillon, M., et al. 2010, ApJ, 709, 159 Baglin, A., Auvergne, M., Boisnard, L., et al. 2006, 36th COSPAR Scientific Assembly, 36, 3749

Bakos, G., Noyes, R. W., Kovács, G., et al. 2004, PASP, 116, 266

Baraffe, I., Chabrier, G., \& Barman, T. 2008, A\&A, 482, 315

Baraffe, I., Chabrier, G., \& Barman, T. 2010, Rep. Prog. Phys., 73, 016901

Baranne, A., Queloz, D., Mayor, M., et al. 1996, A\&AS, 119, 373

Barnes, S. A. 2007, ApJ, 669, 1167

Batygin, K., \& Stevenson, D. J. 2010, ApJ, 714, L238

Bayliss, D., Zhou, G., Penev, K., et al. 2013, AJ, 146, 113

Bernal, J. 1988, National Institute of Standards and Technology Technical Note, 1252

Bodenheimer, P., Hubickyj, O., \& Lissauer, J. J. 2000, Icarus, 143, 2

Borucki, W. J., Koch, D., Basri, G., et al. 2010, Science, 327, 977

Bruntt, H., Bedding, T. R., Quirion, P.-O., et al. 2010a, MNRAS, 405, 1907

Bruntt, H., Deleuil, M., Fridlund, M., et al. 2010b, A\&A, 519, A51

Burrows, A., Hubeny, I., Budaj, J., \& Hubbard, W. B. 2007, ApJ, 661, 502

Cameron, A. C. 2011, in Proc. IAU Symp. 276, eds. A. Sozzetti, M. G. Lattanzi, \& A. P. Boss, 129

Charbonneau, D., Brown, T. M., Latham, D. W., \& Mayor, M. 2000, ApJ, 529, L45

Claret, A. 2000, A\&A, 363, 1081

Claret, A. 2004, A\&A, 428, 1001

Collier Cameron, A., Pollacco, D., Street, R. A., et al. 2006, MNRAS, 373, 799

Collier Cameron, A., Wilson, D. M., West, R. G., et al. 2007, MNRAS, 380, 1230

Collier Cameron, A., Guenther, E., Smalley, B., et al. 2010, MNRAS, 407, 507

Dall, T. H., Santos, N. C., Arentoft, T., Bedding, T. R., \& Kjeldsen, H. 2006, A\&A, 454, 341

Delaunay, B. 1934, Otdelenie Matematicheskikh i Estestvennykh Nauk, 7, 793

Demarque, P., Woo, J., Kim, Y., \& Yi, S. K. 2004, ApJ, 155, 667

Enoch, B., Collier Cameron, A., Parley, N. R., \& Hebb, L. 2010, A\&A, 516, A33

Fortney, J. J., \& Nettelmann, N. 2010, Space Sci. Rev., 152, 423

Fortney, J. J., Marley, M. S., \& Barnes, J. W. 2007, ApJ, 659, 1661

Gardner, J. P., Mather, J. C., Clampin, M., et al. 2006, Space Sci. Rev., 123, 485

Gillon, M., Smalley, B., Hebb, L., et al. 2009, A\&A, 496, 259

Gillon, M., Jehin, E., Magain, P., et al. 2011, in Detection and Dynamics of Transiting Exoplanets, EPJ Web Conf., 11, 6002

Girardi, L., Bressan, A., Bertelli, G., \& Chiosi, C. 2000, A\&AS, 141, 371

Girardi, L., Williams, B. F., Gilbert, K. M., et al. 2010, ApJ, 724, 1030

Gómez Maqueo Chew, Y., Faedi, F., Cargile, P., et al. 2013, ApJ, 768, 79

Gray, D. F. 2008, The Observation and Analysis of Stellar Photospheres, (Cambridge: Cambridge University Press)

Guillot, T., Burrows, A., Hubbard, W. B., Lunine, J. I., \& Saumon, D. 1996, ApJ, 459, L35

Guillot, T., Santos, N. C., Pont, F., et al. 2006, A\&A, 453, L21

Hartman, J. D., Bakos, G. Á., Torres, G., et al. 2011, ApJ, 742, 59

Hebb, L., Collier Cameron, A., Loeillet, B., et al. 2009, ApJ, 693, 1920

Hebrard, G., Collier Cameron, A., Brown, D. J. A., et al. 2013, A\&A, 549, A134

Holmes, S., Kolb, U., Haswell, C. A., et al. 2011, PASP, 123, 1177

Jackson, B., Greenberg, R., \& Barnes, R. 2008, ApJ, 681, 1631

Jehin, E., Gillon, M., Queloz, D., et al. 2011, The Messenger, 145, 2

Kovács, G., Zucker, S., \& Mazeh, T. 2002, A\&A, 391, 369

Kovács, G., Bakos, G., \& Noyes, R. W. 2005, MNRAS, 356, 557

Laughlin, G., Crismani, M., \& Adams, F. C. 2011, ApJ, 729, L7

Leconte, J., Chabrier, G., Baraffe, I., \& Levrard, B. 2010, A\&A, 516, A64

Lendl, M., Anderson, D. R., Collier Cameron, A., et al. 2012, A\&A, 544, A72

Lucy, L. B., \& Sweeney, M. A. 1971, AJ, 76, 544

Magain, P. 1984, A\&A, 134, 189

Mandel, K., \& Agol, E. 2002, ApJ, 580, L171

Marigo, P., Girardi, L., Bressan, A., et al. 2008, A\&A, 482, 883

Maxted, P. F. L., Anderson, D. R., Collier Cameron, A., et al. 2011, PASP, 123, 547

Mayor, M., \& Queloz, D. 1995, Nature, 378, 355

McCullough, P. R., Stys, J. E., Valenti, J. A., et al. 2005, PASP, 117, 783

Pál, A., \& Bakos, G. Á. 2006, PASP, 118, 1474

Pepe, F., Mayor, M., Galland, F., et al. 2002, A\&A, 388, 632

Pepper, J., Pogge, R. W., DePoy, D. L., et al. 2007, PASP, 119, 923

Perna, R., Heng, K., \& Pont, F. 2012, ApJ, 751, 59

Pietrinferni, A., Cassisi, S., Salaris, M., \& Castelli, F. 2004, ApJ, 612, 168

Pollacco, D. L., Skillen, I., Collier Cameron, A., et al. 2006, PASP, 118, 1407

Pollacco, D., Skillen, I., Collier Cameron, A., et al. 2008, MNRAS, 385, 1576

Queloz, D., Eggenberger, A., Mayor, M., et al. 2000, A\&A, 359, L13

Queloz, D., Henry, G. W., Sivan, J. P., et al. 2001, A\&A, 379, 279

Seager, S., \& Mallén-Ornelas, G. 2003, ApJ, 585, 1038 
Y. Gómez Maqueo Chew et al.: Discovery of WASP-65b and WASP-75b

Sestito, P. \& Randich, S. 2005, A\&A, 442, 615

Showman, A. P., \& Guillot, T. 2002, A\&A, 385, 166

Smith, T. C., Henden, A. \& Terrell, D. 2010, in Proc. SAS, 29, 45

Sozzetti, A., Torres, G., Charbonneau, D., et al. 2007, ApJ, 664, 1190

Spiegel, D. S., \& Burrows, A. 2013, ApJ, 772, 76

Stetson, P. B. 1987, PASP, 99, 191

Tamuz, O., Mazeh, T., \& Zucker, S. 2005, MNRAS, 356, 1466

Tinetti, G., Beaulieu, J. P., Henning, T., et al. 2012, Exp. Astron., 34,
Toner, C. G., \& Gray, D. F. 1988, ApJ, 334, 1008

Torres, G., Andersen, J., \& Giménez, A. 2010, A\&ARv, 18, 67

Udalski, A., Paczynski, B., Zebrun, K., et al. 2002, Acta Astron., 52, 1

Valenti, J. A., \& Fischer, D. A. 2005, ApJS, 159, 141

VandenBerg, D. A., Bergbusch, P. A., \& Dowler, P. D. 2006, ApJS, 162, 375

Wu, Y., \& Lithwick, Y. 2013, ApJ, 763, 13

Zacharias, N., Finch, C. T., Girard, T. M., et al. 2012, VizieR Online Data Catalog, I/322 